\title{
Stable Difference Schemes with Uneven Mesh Spacings*
}

\author{
By Melvyn Ciment
}

\begin{abstract}
We consider a finite-difference approximation to the Cauchy problem for a firstorder hyperbolic partial differential equation using different mesh spacings in different portions of the domain. By reformulating our problem as a difference approximation to an initial-boundary value problem, we are able to use the theory of $\mathrm{H}$. O. Kreiss and S. Osher to analyze the $L_{2}$ stability of our scheme.
\end{abstract}

0. Introduction. In solving partial differential equations by finite-difference approximations, there are situations where the solution exhibits large gradients in a localized region. In such problems, one might wish to employ variable mesh patterns. The principal problem is then to obtain consistent difference equations at the interface of the mesh patterns without introducing any type of instability or without loss of overall computational efficiency.

This paper considers the $L_{2}$ stability of a finite-difference solution to the Cauchy problem $u_{t}=A u_{x}+B u_{y}: u(x, y, 0)=\phi(x, y)$, using two different spatial mesh patterns. Problems of this type shall be referred to as mesh refinement problems in this paper.

Stability is analyzed by reformulating our problem as a difference approximation to an initial-boundary value problem for a system of partial differential equations. General sufficient conditions for the stability of such systems are due to H. O. Kreiss [3], [4] and S. Osher [6].

Numerical results obtained on New York University's CDC-6600 are presented which yield evidence of stability for cases not analytically treated (e.g. refinements having corners, and refinements using different time steps in different portions of the domain).

I. Formulation of Problem. We shall refer to the following initial-value problem as Example I.

$$
u_{t}=\alpha u_{x}: u(x, 0)=\phi(x)
$$

for $t>0$ and all $x$ with $\alpha$ constant and $u$ and $\phi$ scalar functions.

Consider a numerical solution using two different patterns of mesh spacings. On the right-hand side of the origin, the mesh length is $\Delta x_{1}$; on the left-hand side, let the spatial mesh size be $\Delta x_{2}$. Setting $p=\Delta x_{1} / \Delta x_{2}$, take the mesh on the right-hand side as more refined than on the left, i.e. $0<p<1$. In the following, let $w_{i}^{n}$ be the

\footnotetext{
Received March 30, 1970.

AMS 1970 subject classifications. Primary 65M05, 65M10; Secondary 65N10.

Key words and phrases. Difference methods, stability, mixed initial-boundary value problems, mesh refinement.

* This research was supported by A.E.C. Contract No. AT(30-1)-1480.
} 
Lax-Wendroff difference approximation [5] to the solution $u(x, t)$ of Example I at $x=j \Delta x_{1}, t=n \Delta t$. Then, for $j=1,2, \cdots$, and $n=0,1,2, \cdots$,

$$
w_{i}^{n+1}=\left(\frac{b_{1}^{2}-b_{1}}{2}\right) w_{i-1}^{n}+\left(1-b_{1}^{2}\right) w_{i}^{n}+\left(\frac{b_{1}^{2}+b_{1}}{2}\right) w_{i+1}^{n}, \quad b_{1}=\alpha \Delta t / \Delta x_{1} .
$$

To treat this initial-value problem by reduction to an initial-boundary value problem in the quarter plane, it is convenient to introduce a new difference function $v_{i}^{n}$ which denotes an approximation to $u\left(-(j-1) \Delta x_{2}, n \Delta t\right)$ for $j \geqq 0$.

Now, with the vector notation

$$
Z_{i}^{n}=\left(\begin{array}{c}
w_{i}^{n} \\
\boldsymbol{v}_{i}^{n}
\end{array}\right), \quad j=0,1,2, \cdots, \quad n=0,1,2, \cdots
$$

and with the obvious definition $w_{0}^{n} \equiv v_{1}^{n}$, the mesh refinement scheme can be expressed as

$$
Z_{i}^{n+1}=\left(\begin{array}{cc}
\frac{b_{1}^{2}-b_{1}}{2} & 0 \\
0 & \frac{b_{2}^{2}+b_{2}}{2}
\end{array}\right) Z_{i-1}^{n}+\left(\begin{array}{cc}
1-b_{1}^{2} & 0 \\
0 & 1-b_{2}^{2}
\end{array}\right) Z_{i}^{n}+\left(\begin{array}{cc}
\frac{b_{1}^{2}+b_{1}}{2} & 0 \\
0 & \frac{b_{2}^{2}-b_{2}}{2}
\end{array}\right) Z_{i+1}^{n}
$$

for $j=1,2, \cdots, n=0,1,2, \cdots$ (where $b_{2}=p b_{1}$ ). Actually, there remains to prescribe an approximation to $u(0, t)$ at each time step, since a formula for $v_{0}^{n}$ is still lacking. To derive an interface condition, we make the natural requirement that our difference scheme be of second-order accuracy at the point $x=0$. Using the consistency conditions derived from Taylor's theorem, one could obtain directly a three-point asymmetrical scheme up to second-order accuracy. However, we prefer an equivalent formulation which expresses $v_{0}^{n}$ by second-order extrapolation in terms of $w_{1}^{n}, v_{1}^{n}$ and $v_{2}^{n}$. We complete the formulation of the mesh refinement problem as a matrix difference approximation to an initial-boundary value problem by expressing this interface condition in the matrix form,

$$
Z_{0}^{n}=\left(\begin{array}{l}
w_{0}^{n} \\
v_{0}^{n}
\end{array}\right)=\left(\begin{array}{cc}
0 & 1 \\
\alpha_{1} & \alpha_{2}
\end{array}\right)\left(\begin{array}{l}
w_{1}^{n} \\
v_{1}^{n}
\end{array}\right)+\left(\begin{array}{ll}
0 & 0 \\
0 & \alpha_{3}
\end{array}\right)\left(\begin{array}{l}
w_{2}^{n} \\
v_{2}^{n}
\end{array}\right), \quad n=0,1,2, \cdots,
$$

where

$$
\alpha_{1}=\frac{2}{p(1+p)}, \quad \alpha_{2}=\frac{2(p-1)}{p}, \quad \alpha_{3}=\frac{1-p}{1+p} .
$$

Because our difference equations were obtained by reflection of the left-hand side, (1.4) is consistent with the hyperbolic system

$$
\left(\begin{array}{l}
w \\
v
\end{array}\right)_{t}=\left(\begin{array}{cc}
\alpha & 0 \\
0 & -\alpha p
\end{array}\right)\left(\begin{array}{l}
w \\
v
\end{array}\right)_{x}
$$

with compatability conditions $w(0, t)=v(0, t)$. We shall refer to the system of difference equations $(1.4 \mathrm{a}, \mathrm{b})$ as System $\mathrm{I}$.

For initial-boundary value problems of the type formulated above, Kreiss [3], 
[4] and Osher [6] have determined algebraic conditions which when satisfied guarantee stability of our difference approximation.

In the algebraic verification of stability, for most of our mesh refinement problems, the following immediate corollary of the Main Theorem and Lemma 3 of Kreiss [4] has been used repeatedly. It is assumed that the reader is familiar with the terminology of that paper.

COROLLARY I. The approximation (B) is stable if

1. Assumptions 1-4 (of [4]) are satisfied, and if

2. for all $z,|z| \geqq 1$, Det $E\left(\tau_{i}(z)\right)=0$ implies that some $l+1$ characteristic roots have absolute value equal to one.

Note, $E\left(\tau_{1}(z)\right)$ refers to the system (1.12) for $|z| \geqq 1$ and for $z=1$ to (1.12') both of [4]. Also note that, for our System $\mathrm{I}, l=1$.

II. Stable Mesh Refinement Schemes. In this section, we verify stability for the mesh refinement problem System I and introduce generally applicable interface conditions which yield stable mesh refinement schemes.

THEOREM I. System I is a stable approximation for the Cauchy problem of Example I for $0<\left|b_{1}\right|<1$.

Proof. For $\left|b_{1}\right|<1$, assumptions 1-4 are satisfied since the Lax-Wendroff scheme is a stable, dissipative scheme. To check stability, find all eigenvalues $\lambda$, of the mesh refinement problem, by expressing the eigensolutions as

$$
Z_{i}^{n}=\lambda^{n} g_{i} ; \quad \sum_{i=1}^{\infty}\left|g_{i}\right|^{2}<\infty ; \quad \lambda \neq 1, \quad|\lambda| \geqq 1,
$$

where $g_{i}=g_{i}(\lambda)$ is determined using the general solution of the characteristic equations

$$
\lambda=\left(\frac{b_{i}^{2}+(-1)^{i} b_{i}}{2}\right) \frac{1}{\tau_{i}}+\left(1-b_{i}^{2}\right)+\left(\frac{b_{i}^{2}+(-1)^{i+1} b_{i}}{2}\right) \tau_{i}, \quad i=1,2 .
$$

The $g_{i}$ take the form

$$
g_{j}(\lambda)=g_{j}=\left[\begin{array}{ll}
\beta_{1} & \tau_{1}^{i} \\
\beta_{2} & \tau_{2}^{j}
\end{array}\right], \quad \beta_{i} \text { constants }
$$

since each $\tau_{i}$ (the characteristic roots) are separated by the unit circle for $|\lambda| \geqq 1$, $\lambda \neq 1$. The $\beta_{i}$ here are referred to as $\sigma=\left(\sigma_{1} \sigma_{2}\right)^{\prime}$ in [4]. We now show that all nontrivial solutions of the form (2.1), when substituted into the boundary condition (1.4b), imply that $\tau_{1}=\tau_{2}=1$. Thus, stability follows by Corollary I.

Substitution of (2.1) into the boundary condition yields

$$
\begin{aligned}
& \text { (a) } \beta_{1}=\beta_{2} \tau_{2} \text {, } \\
& \text { (b) } \beta_{2}=\alpha_{1} \beta_{1} \tau_{1}+\alpha_{2} \beta_{2} \tau_{2}+\alpha_{3} \beta_{2} \tau_{2}^{2} \text {, }
\end{aligned}
$$

for a nontrivial solution, $\beta_{2} \neq 0$. Eliminating $\beta_{1}$ and $\beta_{2}$ gives

$$
p(1-p) \tau_{2}^{2}+2 \tau_{1} \tau_{2}+2\left(p^{2}-1\right) \tau_{2}-p(1+p)=0 .
$$

Equating the expressions for $\lambda$ from (2.2), yields, upon clearing fractions,

$$
\begin{aligned}
2 b_{1}^{2}\left(1-p^{2}\right) \tau_{1} \tau_{2}+\tau_{1}\left[p^{2} b_{1}^{2}+p b_{1}+\left(p^{2} b_{1}^{2}-p b_{1}\right) \tau_{2}^{2}-\right. & \left.\left(b_{1}^{2}+b_{1}\right) \tau_{1} \tau_{2}\right] \\
& -\left(b_{1}^{2}-b_{1}\right) \tau_{2}=0 .
\end{aligned}
$$


Solve for $\tau_{1} \tau_{2}$ and $\tau_{1}$ from (2.4) and substitute into the above to find, after some rearrangement,

$$
\left(\frac{b_{1}^{2}-b_{1}}{2}\right) p^{2}\left(p^{2}-1\right)\left(\tau_{2}-1\right)^{4}=0
$$

For a reasonable problem, this can only be satisfied if $\tau_{2}=1$. Now, setting $\tau_{2}=1$ in (2.4) yields that $\tau_{1}=1$. Q.E.D.

We now present two different interface conditions whose stability is easily demonstrated and which are useful in higher dimensions. Consider the formula for extrapolation of a sufficiently smooth function $v(x)$,

$$
v_{0}=\sum_{i=1}^{m}(-1)^{i+1}\left(\begin{array}{c}
m \\
j
\end{array}\right) v_{i},
$$

where $v_{i}=v(j \Delta x)$ (see e.g. [2]). Alter the format of System I so that $v_{i}^{n} \sim$ $u\left(-j \Delta x_{2}, n \Delta t\right)$, for $j=0,1,2, \cdots$; and so $v_{0}^{n}=w_{0}^{n}$ for all $n$. Using (2.6) to define $v_{0}^{n}$, refer to the resulting mesh refinement approximation scheme as System II.

THEOREM II. System II is a stable approximation to Example I when $\alpha<0$. If $\alpha>0$, then replacing $v_{i}^{n}$ in (2.6) by $w_{i}^{n}$ results in a stable mesh refinement scheme for $\left|b_{1}\right| \leqq 1$.

Proof. The algebraic verification of stability now follows trivially in the obvious matrix formulation.

An interface condition, which has the advantage of being stable regardless of the sign of $\alpha$, and is also suitable for the case of higher dimensions, can be had by taking $p=\Delta x_{1} / \Delta x_{2}=1 / M$ where $M$ is an integer. Assuming a definition for $v_{i}^{n}$ and $w_{i}^{n}$ as in System I, we see that $v_{0}^{n}$ coincides with $w_{M}^{n}$ for $n=0,1, \cdots$. This suggests use of the coarse mesh difference equation at the interface. This boundary condition in matrix form becomes

$$
\left(\begin{array}{l}
w_{0}^{n} \\
v_{0}^{n}
\end{array}\right]=\left(\begin{array}{ll}
0 & 1 \\
0 & 0
\end{array}\right)\left[\begin{array}{l}
w_{1}^{n} \\
v_{1}^{n}
\end{array}\right]+\left(\begin{array}{ll}
0 & 0 \\
1 & 0
\end{array}\right)\left(\begin{array}{l}
w_{M}^{n} \\
v_{M}^{n}
\end{array}\right], \quad n=0,1,2, \cdots .
$$

We shall refer to the difference approximation formed by using (2.7) in conjunction with the difference equations (1.4a) as System III.

THEOREM III. System III is a stable approximation to Example I for $\left|b_{1}\right| \leqq 1$.

Proof. Again, because of the separation of the roots, to check stability by Kreiss' theorem, try a solution of the form (2.1). Substitution into the boundary condition gives for nontrivial solutions

$$
\beta_{1}=\beta_{2} \tau_{2} \text { and } \beta_{2}=\beta_{1} \tau_{1}^{M} .
$$

A nontrivial solution exists only if $\beta_{1}$ and $\beta_{2}$ are both different from zero. Eliminating the $\beta_{i}$ 's, we find

$$
\tau_{2} \tau_{1}^{M}=1
$$

For a bounded solution, the above implies that $\left|\tau_{2}\right|=\left|\tau_{1}\right|=1$. Thus, stability follows from Corollary I.

We remark that the above formulation can be used to show that the interface conditions of Systems II and III can be used to match any 3-point dissipative difference scheme (even different ones on each side) along an interface [1] and retain stability. 
III. Equations with Two Space Dimensions. Consider the analogous mesh refinement problem for

$$
u_{t}=a u_{x}+b u_{y}, \quad a, b \text { constants, for all } x \text { and } y \text { and } t \geqq 0 .
$$

Let the plane $x=0$ be the interface between two mesh patterns. In the right halfplane, take mesh spacings in the $x$ and $y$ directions to be $\Delta x_{1}$ and $\Delta y_{1}$, respectively. In the left half-plane, denote these respective mesh spacings by $\Delta x_{2}$ and $\Delta y_{2}$. We restrict our attention to the case where $p_{1}=\Delta x_{1} / \Delta x_{2}=1 / N$ and $p_{2}=\Delta y_{1} / \Delta y_{2}=$ $1 / M$, where $N$ and $M$ are positive integers.

Let $W_{i, k}^{n}$ and $V_{i, k}^{n}$ denote approximations to $u\left(j \Delta x_{1}, k \Delta y_{1}, n \Delta t\right)$ and

$$
u\left(-(j-1) \Delta x_{2}, k \Delta y_{2}, n \Delta t\right),
$$

respectively, for $n=0,1,2, \cdots, j=0,1,2, \cdots, k=0, \pm 1, \pm 2, \cdots$ (see Fig. 1). As in the one-dimensional problem, one needs to express $W_{0, k}, V_{0, k}$ as a linear combination of the values at neighboring points. From the definitions of our mesh functions,

$$
V_{0, k}=W_{N, k M}, \quad W_{0, k M}=V_{1, k}, k=0, \pm 1, \pm 2, \cdots .
$$

At each time step, we still have to prescribe $W_{0, k M+i}^{n}$ for $k=0, \pm 1, \pm 2, \cdots, t=$ $1,2, \cdots, M-1$. In general, one could prescribe such points as a linear combination of the $V_{1, i}$ values, say,

$$
W_{0, k M+i}^{n}=\alpha_{i,-t} V_{1, k-t}^{n}+\cdots+\alpha_{i, 0} V_{1, k}^{n}+\cdots+\alpha_{i, s} V_{1, k+s}^{n}
$$

for $s, t$ natural numbers.

Now, consider a mesh refinement problem for a 9-point dissipative approximation to (3.1), (e.g. the Lax-Wendroff scheme [5]). Then

$$
\begin{aligned}
\text { (a) } V_{i, k}^{n+1}= & \sum_{\alpha=-1}^{1} \sum_{\beta=-1}^{1} C_{\alpha, \beta}^{(1)} V_{j+\alpha, k+\beta}^{n}, \\
\text { (b) } W_{i, k}^{n+1}= & \sum_{\alpha=-1}^{1} \sum_{\beta=-1}^{1} C_{\alpha, \beta}^{(2)} W_{j+\alpha, k+\beta}^{n}, \\
& n=0,1,2, \cdots, j=1,2,3, \cdots, k=0, \pm 1, \pm 2, \cdots,
\end{aligned}
$$

where the $C_{\alpha, \beta}^{(i)}$ are functions of $\Delta t, \Delta x_{i}, \Delta y_{i}$. Then, (3.2)-(3.4) is a well-defined approximation to (3.1). Again, we treated the mesh refinement problem as a system of difference equations for an initial-boundary value problem. Because of the more complicated nature of the boundary condition in two dimensions, we found it necessary to introduce a vector of dimension $M+1$ corresponding to the data $\left(V_{i, k}^{n}, W_{i, k M}^{n}, \cdots, W_{j, k M+M-1}^{n}\right)$. Then, reformulating our difference equations consistent with a system of $M+1$ partial differential equations, we are able to prove [1].

THEOREM IV. If the difference schemes (3.4) are stable for the pure Cauchy problem, then the mesh refinement scheme (3.2)-(3.4) is a stable approximation.

Proof. Because of the diagonal structure of our systems, it suffices to study all eigensolutions of the form

$$
Z_{i, k}^{n}=\lambda^{n} y_{0}^{k} g_{i}, \quad\left|y_{0}\right|=1, \quad \sum_{i=1}^{\infty}\left|g_{i}\right|^{2}<\infty,
$$




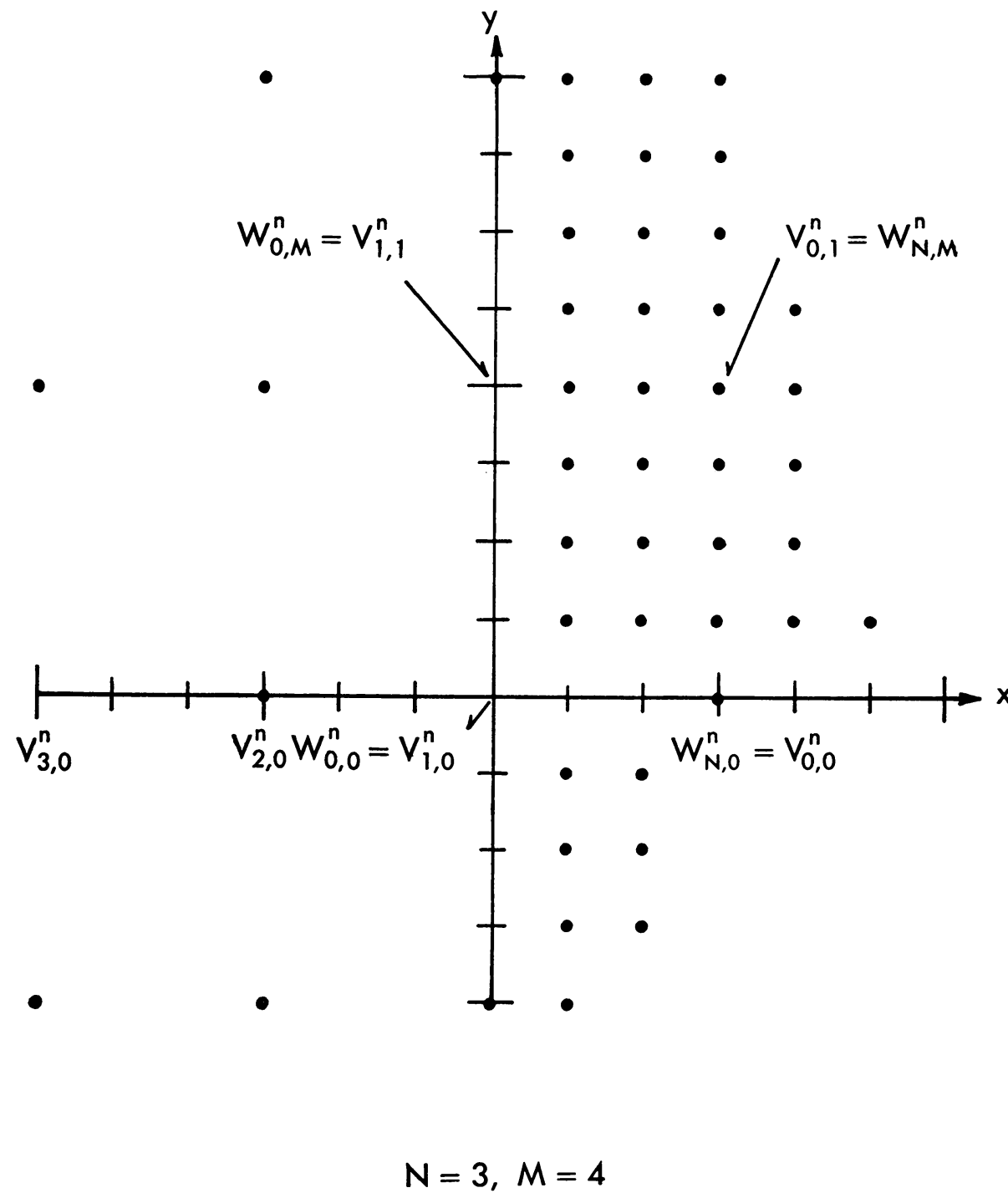

FIGURE 1

and show that Corollary I is satisfied for all $y_{0},\left|y_{0}\right|=1$. One finds that the conditions (3.2), when expressed in the indicated matrix form, lead directly to Eq. (2.8). Thus, arguing as in Theorem III, stability is demonstrated.

We note that following Kreiss [3] and [1], the matrix analogues of Theorems II-IV hold for Lipschitz continuous diagonal systems.

IV. Computational Results. Calculations were performed using the mesh refinement schemes of Theorems I-IV. The primary purpose was to compare the relative accuracy of the different interface conditions discussed above. We tested our methods on problems where the analytical solution was available.

a. One-Dimensional Periodic Case. Table 1 lists the results of several calculations 
using the Lax-Wendroff scheme for the initial-value problem of Example I with $\alpha \equiv 1$ and solution $u(x, t)=\sin 4 \pi(x+t)$. We used the periodicity of our solution to restrict our calculations to the interval $[0,1]$ with refined region $I_{R} \equiv\left\{x: \frac{1}{3} \leqq x \leqq \frac{2}{3}\right\}$. By $e(v)$ and $e(w)$, we denote the maximum error of our scheme from the solution in the unrefined region and the interior of $I_{R}$, respectively.

To describe if any additional error due to our unusual interface conditions propagated into $I_{R}$, we compute a quantity defined by

$$
e_{100} \equiv \max _{j=2,3, \cdots, 100}\left\{\left|u(x, t)-w_{i}^{n}\right|: t=n \Delta t, x=\frac{1}{3}+(j-1) \Delta x_{1}\right\},
$$

since the error from $x \geqq \frac{2}{3}$ cannot reach into more than 400 grid points of $I_{R}$ in 400 time steps.

In the mesh refinement problem, a small time step is needed for stability in $I_{R}$. However, using the same time step in the unrefined region will reduce accuracy there. To rectify this situation we also considered using different time steps in the different regions. The only difficulty in using this approach is at the interface. The interfaces for Systems I and III were treated by first advancing $w_{1}^{n / p+i}$ and $w_{501}^{n / p+i}$, for $i=1,2, \cdots, 10$. Then, the remaining is advanced in the usual manner until all points are at the same time level. Rows 7 and 8 of Table 1 show the improved results, and a comparison of rows 1-3 (or 7,8 ) with row 6 shows that the interface conditions used at $x=\frac{1}{3}$ do not propagate much error into $I_{R}$ since the $e_{100}$ agree to 7 places in all entries.

In Table 2 we present the results of computations performed for Example I with

$$
\begin{aligned}
\alpha=\alpha(x) & =\frac{1}{2}, & & 0 \leqq x \leqq \frac{1}{3}, \\
& =\frac{2+\sin 6 \pi x}{4}, & & x \in I_{R}, \\
& =\frac{1}{2}, & & \frac{2}{3} \leqq x \leqq 1 .
\end{aligned}
$$

The quantities displayed in Table 2 are defined exactly in the same way as in

\begin{tabular}{|c|c|c|c|c|}
\hline & $t$, time & $e(v)$ & $e(w)$ & $e_{100}$ \\
\hline 1) System I & $400 \Delta t$ & $3.332 \times 10^{-3}$ & $1.591 \times 10^{-3}$ & $6.3 \times 10^{-6}$ \\
\hline 2) System II $(m=3)$ & $400 \Delta t$ & $3.332 \times 10^{-3}$ & $1.656 \times 10^{-3}$ & $6.3 \times 10^{-6}$ \\
\hline 3) System III & $400 \Delta t$ & $3.332 \times 10^{-3}$ & $1.629 \times 10^{-3}$ & $6.7 \times 10^{-6}$ \\
\hline 4) No Refinement $\left(\Delta x_{2}\right)$ & $400 \Delta t$ & $3.333 \times 10^{-3}$ & & \\
\hline 5) No Refinement $\left(\Delta x_{2}\right)$ & $40 \Delta t^{\prime}$ & $8.909 \times 10^{-4}$ & & \\
\hline 6) No Refinement $\left(\Delta x_{1}\right)$ & $400 \Delta t$ & $8.9 \times 10^{-6}$ & $8.9 \times 10^{-6}$ & $6.3 \times 10^{-6}$ \\
\hline 7) Uneven Time (I) & $40 \Delta t^{\prime}$ & $8.909 \times 10^{-4}$ & $4.493 \times 10^{-4}$ & $6.3 \times 10^{-6}$ \\
\hline 8) Uneven Time (III) & $40 \Delta t^{\prime}$ & $8.909 \times 10^{-4}$ & $4.493 \times 10^{-4}$ & $6.3 \times 10^{-6}$ \\
\hline
\end{tabular}
Table 1.

\section{TABLE 1}

$$
\begin{gathered}
\alpha \equiv 1 \\
\Delta t=1 / 1750 ; \quad \Delta t^{\prime}=1 / 175 ; \quad \Delta x_{2}=1 / 150 ; \quad \Delta x_{1}=1 / 1500
\end{gathered}
$$


TABLE 2

$$
\begin{gathered}
\alpha=\alpha(x) \text { as in }(4.1) \\
\Delta t=1 / 1250 ; \quad \Delta t^{\prime}=1 / 125 ; \quad \Delta x_{2}=1 / 150 ; \quad \Delta x_{1}=1 / 1500
\end{gathered}
$$

\begin{tabular}{lrccc}
\hline & $t$, time & $e(v)$ & $e(w)$ & $e_{100}$ \\
\cline { 2 - 5 } & $400 \Delta t$ & $7.136 \times 10^{-3}$ & $1.397 \times 10^{-3}$ & $1.000 \times 10^{-3}$ \\
1) System I & $400 \Delta t$ & $7.134 \times 10^{-3}$ & $2.764 \times 10^{-3}$ & $1.000 \times 10^{-3}$ \\
2) System II $(m=3)$ & $400 \Delta t$ & $9.993 \times 10^{-3}$ & $1.401 \times 10^{-3}$ & $1.000 \times 10^{-3}$ \\
3) System III & $400 \Delta t$ & $1.180 \times 10^{-2}$ & & \\
4) No Refinement $\left(\Delta x_{2}\right)$ & $40 \Delta t^{\prime}$ & $2.358 \times 10^{-2}$ & & \\
5) No Refinement $\left(\Delta x_{2}\right)$ & $27 \Delta t^{*}$ & $2.901 \times 10^{-2}$ & & \\
6) No Refinement $\left(\Delta x_{2}\right)$ & & & & \\
$\quad \Delta t^{*}=15 / 1250$ & $40 \Delta t^{\prime}$ & $7.668 \times 10^{-3}$ & $1.652 \times 10^{-3}$ & $1.000 \times 10^{-3}$ \\
7) Uneven Time (I) & $400 \Delta t$ & & & \\
& $40 \Delta t^{\prime}$ & $7.668 \times 10^{-3}$ & $1.611 \times 10^{-3}$ & $1.000 \times 10^{-3}$ \\
8) Uneven Time (III) & $400 \Delta t$ & & & \\
9) Uneven Time (III) & $27 \Delta t^{*}$ & $4.841 \times 10^{-3}$ & $1.553 \times 10^{-3}$ & $1.086 \times 10^{-3}$ \\
& $405 \Delta t$ & & & \\
\hline
\end{tabular}

b. Two-Dimensional Periodic Case. Calculations were performed for the initialvalue problem $u_{t}=-u_{x}+u_{y}$ with periodic solution $u(x, t)=\sin 2 \pi(x-t)$ $\cdot \cos 2 \pi(y+t)$ restricted to the unit square $0 \leqq x, y \leqq 1$. The refined region was taken as $D_{R}=\left\{(x, y): \frac{1}{3} \leqq x, y \leqq \frac{2}{3}\right\}$. As in Table 1 , we describe the accuracy with the analogous quantities $e(v)$ and $e(w)$, the maximum errors of our scheme in the unrefined region and $D_{R}$, respectively. To describe the accuracy of the refined scheme away from the interfaces, we compute the error in the interior of $D_{R}, e_{i n 1}$ as the maximum error in the interior square $\left\{(x, y): \frac{4}{9} \leqq x, y \leqq \frac{5}{9}\right\}$. Table 3 lists the results of our calculations using the Lax-Wendroff scheme with our mesh refinement scheme

TABLE 3

$$
\Delta t=1 / 1750 ; \quad \Delta x_{2}=\Delta y_{2}=1 / 45 ; \quad \Delta x_{1}=\Delta y_{1}=1 / 225
$$

\begin{tabular}{lrccc}
\hline & $t$, time & $e(v)$ & $e(w)$ & $e_{\mathrm{int}}$ \\
\cline { 2 - 5 } 1) Ordinary refinement & $100 \Delta t$ & $1.182 \times 10^{-3}$ & $1.143 \times 10^{-3}$ & $4.36 \times 10^{-5}$ \\
2) No refinement & & & & \\
$\left(\Delta x_{2}, \Delta y_{2}\right)$ & & & & \\
a) $\Delta t=1 / 1750$ & $100 \Delta t$ & $1.165 \times 10^{-3}$ & & \\
b) $\Delta t^{\prime}=5 / 1750$ & $20 \Delta t^{\prime}$ & $1.104 \times 10^{-3}$ & & \\
c) $\Delta t^{*}=1 / 175$ & $10 \Delta t^{*}$ & $8.895 \times 10^{-4}$ & & \\
3) Uneven time & $20 \Delta t^{\prime}$ & $1.104 \times 10^{-3}$ & $1.033 \times 10^{-3}$ & $4.36 \times 10^{-5}$ \\
& $=100 \Delta t$ & & & \\
4) Uneven time & $10 \Delta t^{*}$ & $8.895 \times 10^{-4}$ & $7.822 \times 10^{-4}$ & $4.36 \times 10^{-5}$ \\
& $=100 \Delta t$ & & & \\
\hline
\end{tabular}


using centered third-order polynomial interpolation along the interface. This is compared with ordinary Lax-Wendroff with three choices of time steps. We then compute with these time steps, using uneven time steps in a manner similar to the one-dimensional case. Our computations show that the interface condition does not introduce any additional errors, and the error in the unrefined region compares with the error for ordinary no refinement.

Acknowledgements. The author wishes to express his gratitude to Professor Peter D. Lax for suggesting the subject of this investigation, and for his continued interest, guidance, and encouragement, while this work was being completed.

The author is also grateful to Professor Heinz O. Kreiss for offering many valuable suggestions. Thanks are also due to Professor Stanley Osher for several interesting discussions, and to Professor Olof Widlund for his helpful comments on the original manuscript.

This is part of a dissertation [1] in mathematics submitted to the faculty of the Graduate School of New York University in partial fulfillment of the requirements for the degree of Doctor of Philosophy.

Department of Mathematics

University of Michigan

Ann Arbor, Michigan 48104

1. M. CIMENT, Stable Difference Schemes With Uneven Mesh Spacings, A.E.C. Research and Development Report \#NYO-1480-100, Ph.D. Thesis, New York University, New York, 1968.

2. E. IsaAcson \& H. B. Keller, Analysis of Numerical Methods, Wiley, New York, 1966. MR 34 \# 924.

3. H. O. KREISS, Difference Approximations for Initial-Boundary Value Problems for Hyperbolic Differential Equations, Proc. Adv. Sympos. Numerical Solutions of Partial Differential Equations (Madison, Wis., 1965), Wiley, New York, 1966, pp. 141-166. MR 35 \#5156.

4. H. O. KREISS, "Stability theory for difference approximations of mixed initial boundary value problems. I," Math. Comp., v. 22, 1968, pp. 703-714. MR 39 \#2355.

5. P. D. LAX \& B. WENDROFF, "Difference schemes for hyperbolic equations with higher order accuracy," Comm. Pure Appl. Math., v. 17, 1964, 381-398. MR 30 \#722.

6. S. OsHER, "Systems of difference equations with general homogeneous boundary conditions," Trans. Amer. Math. Soc., v. 137, 1969, pp. 177-201. MR 38 \#6259.

7. R. D. RIChtMYer \& K. W. Morton, Difference Methods for Initial Value Problems, 2nd ed., Interscience Tracts in Pure and Appl. Math., no. 4, Interscience, New York, 1967. MR 36 \#3515. 